UDC 530.19

\title{
Gluon field distribution between three infinitely spaced quarks
}

\author{
Dzhunushaliev V. \\ Al-Farabi Kazakh National University, IETP, Almaty 050040, Kazakhstan
}

\begin{abstract}
The gluon field distribution between three infinitely spaced quarks is obtained. The field distribution between every pair is approximated by an infinite flux tube filled with a color longitudinal electric field. The origin of the color electric field is a nonlinear term in the field strength of nonabelian gauge field. Such construction can be considered as a rough model of three quarks in a nucleon.
\end{abstract}

Keywords: field distribution, quarks, flux tube.

PACS number(s): 11.15.Tk; 12.90.+b; 14.70.Dj

\section{Introduction}

Non-abelian gauge theories are central to our current understanding of physical phenomena (excluding gravity). The perturbative analysis of such theories is fairly well understood by now, having been extensively developed over the last three decades. Many of the nonperturbative aspects are also more or less understood at a qualitative level. However, it is fair to say that, as of now, we do not have calculational techniques or detailed understanding regarding nonperturbative phenomena in nonAbelian gauge theories.

The perturbative description of elementary particles is essentially based on the field-particle duality which means that each field in a quantum field theory is associated with a physical particle. On the other hand, the situation in QCD is more complicated, however. For a description of confinement of quarks and gluons within the framework of local quantum field theory, the elementary fields have to be divorced completely from a particle interpretation[1].

Therefore, to study the infrared behavior of QCD amplitudes non-perturbative methods are required. One promising approach to non-perturbative phenomena in QCD is provided by studies of truncated systems of its Dyson - Schwinger equations, the equations of motion for QCD Green's functions. The underlying conjecture to justify such a truncation of the originally infinite set of Dyson Schwinger equations is that a successive inclusion

* Corresponding author: e-mail: v.dzhunushaliev@gmail.com of higher n-point functions in self-consistent calculations will not result in dramatic changes to previously obtained lower $\mathrm{n}$-point functions. To achieve this it is important to incorporate as much independent information as possible in constructing those n-point functions which close the system.

Usually the attempts to solve problems in QCD are based on notion of gluon - a quantum of SU(3) gauge field. Tacitly assuming that quantized field is a cloud of quanta (gluons in QCD). But this is not evident for a quantum field theory with strong interaction where it is necessary to use a nonperturbative quantum field theory. It is possible that strongly interacting quantum fields more similar to a turbulent fluid where is a preferred direction (for example, it can be flux tube stretched between quark and antiquark) and fluctuations around nonzero field there exist. In standard quantum field theory the interaction between quanta happens in a vertex. The vertex is 0 -dimensional object where 0 -dimensional objects -- particles do interact. All it gives rise to the idea that strongly interacting quantum fields can not be described on the language of particles -quanta. Mathematically it means that in this case it is not possible to use Feynman diagram technique to the description of strongly interacting quantum fields. The consequence of this statement is: if we can not use Feynman diagram technique then we can not use the notion of quanta for the description of strongly interacting quantum fields. In other words strongly interacting quantized fields do exist but they do not formed with quanta. For example, quantum gluon field does exist but gluons (in some energy region) do not exist, for gravity this statement is even more strict: quantum gravity does exist but gravitons do not exist. 
One interesting problem in quantum chromodynamics is the calculation of gluon distribution between quarks. If we calculate the field distribution between two quarks we will obtain a hypothesized flux tube that is the main point of the confinement problem in quantum chromodynamics. The problem is analogous to the calculation of a static electric field between electron and positron in electrodynamics with one essential difference. The field distribution between quarks and between electric charges are essentially different: the gluon field between two quarks is confined into a tube but the electric field is distributed in the whole space. If we will calculate the field distribution between three quarks we will obtain a gluon distribution between quarks in a hadron. In electrodynamics we have not any analogous problem because quarks may have three different kind of charge: red, green and blue. But the electric charge has not a color index.

Here we will calculate the gluon field distribution between three infinitely separated quarks. But why infinitely separated? The answer is that we have the field distribution between two quarks [2]. In this case in a first approximation we can obtain the field distribution between three quarks as three flux tubes connecting three infinitely spaced quarks.

The analytical calculations of the gluon field distribution between quarks can not be carried out in the consequence of missing of a nonperturbative calculation technique in quantum field theory. There exist calculations of the field distribution between quarks on lattice, see for example, Ref. [3]. The lattice calculations allow us to investigate a gauge field distribution between quarks. But the problem in lattice calculations is that we have not any analytical expression for investigated quantities such as: fields; potentials; energy, action, monopole densities and so on. Another problem is that we are not self-confident yet that there exists a limit when a grid size of lattice tends zero.

Our calculations are following: (a) we will recall how one can obtain the flux tube following to Ref. [2], (b) we will clarify what is the origin of a color electric field in the flux tube and (c) we will connect three infinitely spaces quarks with these flux tubes filled with color electric fields.

\section{Flux tube}

Nonperturbative quantization is unresolved problem in modern theoretical physics. Here for the calculations in quantum chromodynamics we use following approximate method [4]: we calculate $\mathrm{SU}(3)$ gluon condensate $\left\langle\hat{\mathcal{F}}^{B \mu \nu} \hat{\mathcal{F}}_{\mu \nu}^{B}\right\rangle$ which up to a sign is the quantum averaged $\mathrm{SU}(3)$ Lagrangian

$$
\left\langle\mathcal{L}_{S U(3)}\right\rangle=-\frac{1}{4 g^{2}}\left\langle\hat{\mathcal{F}}^{B \mu \nu} \hat{\mathcal{F}}_{\mu \nu}^{B}\right\rangle
$$

where $\mathcal{F}_{\mu \nu}^{B}=\partial_{\mu} A_{\nu}^{B}-\partial_{\nu} A_{\mu}^{B}+g f^{B C D} A_{\mu}^{C} A_{\nu}^{D}$ is the field strength; $B, C, D=1, \ldots, 8$ are the $\mathrm{SU}(3)$ color indices; $g$ is the coupling constant; $f^{B C D}$ are the structure constants for the $\mathrm{SU}(3)$ gauge group and $g$ is the coupling constant. In Ref. [2] it is shown that using some assumptions and approximations one can reduce $\mathrm{SU}(3)$ Lagrangian (1) to an effective Lagrangian

$$
\begin{aligned}
& \mathcal{L}_{e f f}=-\frac{1}{4 g^{2}} F_{\mu \nu}^{a} F^{a \mu \nu}+\frac{1}{2}\left|\phi_{\mu}\right|^{2}-\frac{\lambda}{4}\left(|\phi|^{2}-\right. \\
& \left.\phi_{\infty}^{2}\right)^{2}++\frac{1}{2} A_{\mu}^{a} A^{a \mu}|\phi|^{2}-\frac{1}{2} m_{a}^{2} A_{\nu}^{a} A^{a \mu}
\end{aligned}
$$

where $\phi$ is a complex scalar field describing quantum fluctuations of $A_{\mu}^{m} \in S U(3) / S U(2)$ gauge field components; $\phi_{\infty}$ and $m_{a}$ are some parameters.

In order to obtain effective Lagrangian (2) we have used following assumptions:

- The first assumption is that the $\mathrm{SU}(3)$ gauge potential $A_{\mu}^{B} \in S U(3), B=1,2, \cdots, 8$ can be separated on two parts:

- the first one is the gauge components $A_{\mu}^{a} \in$ $S U(2) \subset S U(3)$ which is in a classical state;

- the second one is $A_{\mu}^{m} \in S U(3) / S U(2)$ and it is in a quantum state.

One can say that $A_{\mu}^{a}$ is in an ordered phase and $A_{\mu}^{m}$ is in a disordered phase.

- The second assumption is that the 2-point Green's function of color quantum field can be approximately presented as the product of scalar fields with some coefficients having color and Lorentzian indices.

- The third assumption is that the 4-point Green's function can be decomposed as the product of two 2-point Green's functions.

The second and third assumptions gives rise to the fact that 2 and 4-point Green's functions are

$$
\begin{aligned}
& \left(G_{2}\right)_{\mu \nu}^{m n}\left(x_{1}, x_{2}\right)=\left\langle A_{\mu}^{m}\left(x_{1}\right) A_{\nu}^{n}\left(x_{2}\right)\right\rangle \approx \\
& \approx C_{\mu \nu}^{m n} \phi\left(x_{1}\right) \phi^{*}\left(x_{2}\right)+\widetilde{m}_{\mu \nu}^{m n}, \\
& G_{\mu \nu \rho \sigma}^{m n p q}\left(x_{1}, x_{2}, x_{3}, x_{4}\right)= \\
& =\left\langle A_{\mu}^{m}\left(x_{1}\right) A_{\nu}^{n}\left(x_{2}\right) A_{\rho}^{p}\left(x_{3}\right) A_{\sigma}^{q}\left(x_{4}\right)\right\rangle \approx
\end{aligned}
$$




$$
\approx\left(G_{2}\right)_{\mu \nu}^{m n}\left(x_{1}, x_{2}\right)\left(G_{2}\right)_{\rho \sigma}^{p q}\left(x_{1}, x_{2}\right)
$$

The field equations for the Lagrangian (2) are

$$
\begin{gathered}
\frac{1}{4 g^{2}} D_{\nu} F^{a \mu \nu}=\left(|\phi|^{2}-m_{a}^{2}\right) A^{a \mu}, \\
\text { no summation over } a, \\
\phi_{\mu}^{; \mu}=-\lambda \phi\left(|\phi|^{2}-\phi_{\infty}^{2}\right)+A_{\mu}^{a} A^{a \mu} \phi .
\end{gathered}
$$

In Ref. [2] the index $a=1,2,3$, i.e. the $\mathrm{SU}(2)$ subgroup on generators $\lambda_{1,2,3}$ is spanned $\left(\lambda_{B}\right.$ are Gell-Mann matrices that are SU(3) generators). But it is not necessary: the index acan be the index of any $S U(2) \subset S U(3)$ subgroup. For example, it can be:

$$
\begin{gathered}
a=(1,2,3) ;(1,4,7) ;(1,5,6) ;(2,4,6) ;(2,5,7) ; \\
(3,4,5) ;(3,6,7)
\end{gathered}
$$

It follows form the fact there are following $\mathrm{SU}(3)$ structure constants

$$
\begin{gathered}
f_{123}=1, \\
f_{147}=-f_{156}=f_{246}=f_{257}=f_{345}=-f_{367}=\frac{1}{2}
\end{gathered}
$$

that describe $\mathrm{SU}(2)$ subgroups $S U(2) \subset S U(3)$. This difference is unessential because we can redefine coupling constant $g$ in such a way that to kill the factor $f_{a b c}=1 / 2$.

If we choose $\mathrm{SU}(2)$ subgroup spanned on $\mathrm{SU}(3)$ generators $\lambda_{1,4,7}$ then the solution one can search in the following form

$$
A_{t}^{1}(\rho)=f(\rho) ; \quad A_{z}^{4}(\rho)=v(\rho) ; \quad \phi(\rho)=\phi(\rho)
$$

here $z, \rho, \varphi$ are cylindrical coordinate system. The substitution into equations (5)(6) gives us

$$
\begin{gathered}
f^{\prime \prime}+\frac{f^{\prime}}{x}=f\left(\phi^{2}+v^{2}-m_{1}^{2}\right), \\
v^{\prime \prime}+\frac{v \prime}{x}=v\left(\phi^{2}-f^{2}-m_{2}^{2}\right), \\
\phi^{\prime \prime}+\frac{\phi^{\prime}}{x}=\phi\left[-f^{2}+v^{2}+\lambda\left(\phi^{2}-\mu^{2}\right)\right]
\end{gathered}
$$

here we redefined $g \phi / \phi(0) \rightarrow \phi, f / \phi(0) \rightarrow f$, $v / \phi(0) \rightarrow v, \quad g \phi_{\infty} / \phi(0) \rightarrow \mu, \quad g m_{1,2} / \phi(0) \rightarrow$ $m_{1,2}, \rho \sqrt{\phi(0)} \rightarrow x$. The color electric and magnetic fields are

$$
F_{t \rho}^{1}=-f^{\prime}, \quad F_{z \rho}^{4}=-v^{\prime}, \quad F_{t z}^{7}=f v .
$$

The most important here is that the color longitudinal electric field $F_{t z}^{7}$ is not a gradient of a gauge potential but is the consequence of a nonlinear term in the definition of the field tensor $F_{\mu \nu}^{a}$.

For the numerical calculations we choose the following parameters values

$$
\lambda=0.1, \quad \phi(0)=1, \quad v(0)=0.5, \quad f(0)=0.2 .
$$

The numerical values of these parameters are not unique but qualitative behavior of the functions $f(r), v(r), \phi(r)$ are the same. The solution of Eq's (9)-(11) with parameters (13) is presented in figures 1 and 2.

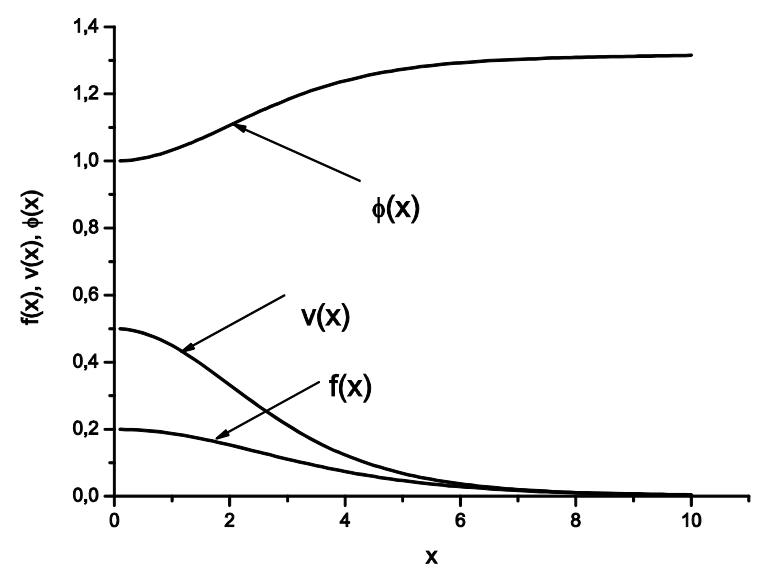

Figure $1-$ The profiles of functions $f^{*}(x), v^{*}(x), \phi^{*}(x)$. 


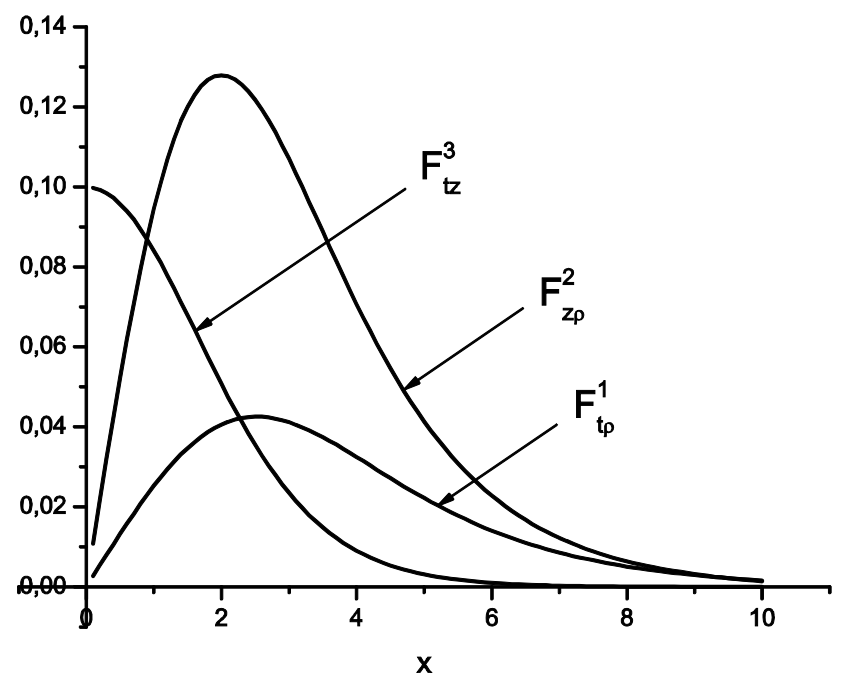

Figure 2 - The profiles of color fields $F_{t \rho}^{1}(x)=-f^{\prime}, F_{z \rho}^{4}(x)=-v^{\prime}, F_{t z}^{7}(x)=f(x) v(x)$.

\section{The sources of longitudinal electric field at the end of the flux tube}

Now we would like to discuss what is the source of the above mentioned longitudinal electric field $F_{t z}^{7}$. Firstly we would like to calculate the flux of the chromoelectric field $F_{t z}^{7}$

$$
\Phi=2 \pi \int_{0}^{\infty} \rho F_{t z}^{3} d \rho \neq 0 .
$$

It is nonzero and consequently at the ends of the color flux tube should be color sources of this color field. For the determination of the color sources we will remind the SU(3) Lagrangian with quarks

$$
\mathcal{L}_{Q C D}=-\frac{1}{2} \mathcal{F}^{B \mu \nu} \mathcal{F}_{\mu \nu}^{B}+\bar{\psi}\left(p_{\mu}+\frac{g}{2} A_{\mu}^{B} \lambda^{B}\right) \psi
$$

here we follow to [5]; $\lambda^{B} / 2$ are the $\mathrm{SU}(3)$ generators; spinor $\psi$ describe quarks

$$
\psi=\left(\begin{array}{l}
\psi_{r} \\
\psi_{g} \\
\psi_{b}
\end{array}\right)
$$

where spinors $\psi_{r, g, b}$ describe red, green and blue quarks correspondingly. The term $\bar{\psi} \lambda^{B} \psi$ describes the source for the $F_{\mu \nu}^{B}$ field.

In our case we have $F_{t z}^{7}(x)$. Consequently the source of the color nonabelian longitudinal electric field has the form

$$
\begin{gathered}
\bar{\psi} \lambda^{7} \psi=\left(\bar{\psi}_{r}, \bar{\psi}_{g}, \bar{\psi}_{b}\right)\left(\begin{array}{ccc}
0 & 0 & 0 \\
0 & 0 & -i \\
0 & i & 0
\end{array}\right)\left(\begin{array}{l}
\psi_{r} \\
\psi_{g} \\
\psi_{b}
\end{array}\right)= \\
=i\left(\bar{\psi}_{b} \psi_{g}-\bar{\psi}_{g} \psi_{b}\right) .
\end{gathered}
$$

Thus at the ends of this flux tube there are quarks $\left(\psi_{r}, \psi_{g}, \psi_{b}\right)^{T}$ and $\psi_{g}, \psi_{b}$ components generate the green/blue color longitudinal electric field $F_{t z}^{7}(x)$.

\section{Flux tubes between three quarks spaced at the infinity}

Now is the time to discuss the simplest model of gluon field distribution between three quarks. We simplify such model moving off the quarks to the infinity. In this case we can use the flux tube obtained in the section 3 . Let us consider three quarks $Q_{1,2,3}$ in figure 3 .

Let us assume that the flux tube with $F_{t z}^{7}(x)$ longitudinal electric field is stretched between $Q_{1}$ and $Q_{2}$ quarks.

Now we change $\mathrm{SU}(2)$ subgroup spanned on $\lambda_{1,4,7}$ on the $\mathrm{SU}(2)$ subgroup spanned on $\lambda_{1,5,6}$. Then one can use ansatz

$$
A_{t}^{1}(\rho)=f(\rho) ; \quad A_{z}^{6}(\rho)=v(\rho) ; \quad \phi(\rho)=\phi(\rho) .
$$




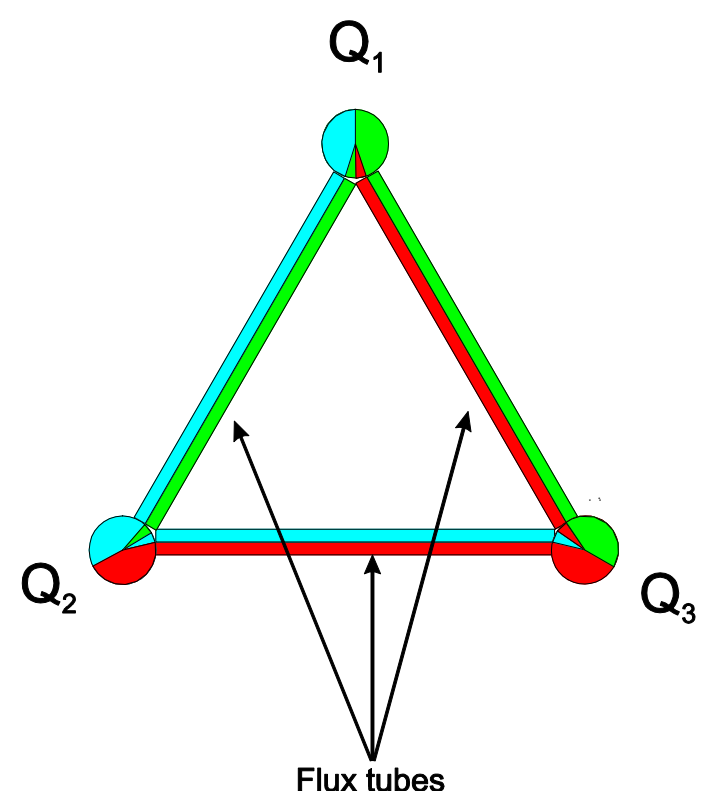

Figure 3 - Three infinitely spaced quarks connected with color flux tubes.

For such choice the color electric and magnetic fields will be

$$
F_{t \rho}^{1}=-f^{\prime}, \quad F_{z \rho}^{6}=-v^{\prime}, \quad F_{t z}^{5}=f v .
$$

Consequently the source of the color longitudinal electric has the form

$$
\begin{gathered}
\bar{\psi} \lambda^{5} \psi=\left(\bar{\psi}_{r}, \bar{\psi}_{g}, \bar{\psi}_{b}\right)\left(\begin{array}{ccc}
0 & 0 & -i \\
0 & 0 & 0 \\
i & 0 & 0
\end{array}\right)\left(\begin{array}{l}
\psi_{r} \\
\psi_{g} \\
\psi_{b}
\end{array}\right)= \\
=i\left(\bar{\psi}_{b} \psi_{r}-\bar{\psi}_{r} \psi_{b}\right) .
\end{gathered}
$$

Thus at the ends of this flux tube there are quarks $Q_{2,3}=\left(\psi_{r}, \psi_{g}, \psi_{b}\right)^{T}$ and their $\psi_{r}, \psi_{b}$ components generate the red/blue color longitudinal electric field $F_{t z}^{5}(x)$.

In the same way one can choose the SU(2) subgroup spanned on $\mathrm{SU}(3)$ generators $\lambda_{4,6,2}$. Then

$$
A_{t}^{4}(\rho)=f(\rho) ; \quad A_{z}^{6}(\rho)=v(\rho) ; \quad \phi(\rho)=\phi(\rho) .(21)
$$

For such choice the color electric and magnetic fields will be

$$
F_{t \rho}^{4}=-f^{\prime}, \quad F_{z \rho}^{6}=-v^{\prime}, \quad F_{t z}^{2}=f v .
$$

Consequently the source of the color longitudinal electric has the form

$$
\begin{gathered}
\bar{\psi} \lambda^{2} \psi=\left(\bar{\psi}_{r}, \bar{\psi}_{g}, \bar{\psi}_{b}\right)\left(\begin{array}{ccc}
0 & -i & 0 \\
i & 0 & 0 \\
0 & 0 & 0
\end{array}\right)\left(\begin{array}{l}
\psi_{r} \\
\psi_{g} \\
\psi_{b}
\end{array}\right)= \\
i\left(\bar{\psi}_{g} \psi_{r}-\bar{\psi}_{r} \psi_{g}\right) .
\end{gathered}
$$

Thus at the ends of this flux tube there are quarks $Q_{1,3}=\left(\psi_{r}, \psi_{g}, \psi_{b}\right)^{T}$ and their $\psi_{r}, \psi_{g}$ components generate the red/green color longitudinal electric field $F_{t z}^{2}(x)$.

Finally, connecting three quarks $Q_{1,2,3}$ with corresponding color flux tubes we will obtain the construction from three quarks presented in fig. 3 .

\section{Conclusions and discussion}

Thus we have obtained the field distribution of the SU(3) gauge field between three infinitely spaced quarks, see fig. 3. In order to obtain such distribution we have used the flux tube solution obtained by a nonperturbative quantization method [4].

The problem of obtaining the field distribution between three color charges has not any analogy to a similar problem in electrodynamics. The point is that the 
color charges have additional degrees of freedom: color indices and this fact gives rise to principal difference between nonabelian and abelian gauge fields. The difference can be illustrated for the field distribution for three $\mathrm{SU}(3)$ color charges - $r$, g, b quarks. Let us consider fig. 3. For every pair $Q_{i}, Q_{j}$ the gluon field is concentrated in a tube (in the first approximation). A color longitudinal electric field is created from the pair either $\mathrm{r}, \mathrm{g}$ or $\mathrm{g}, \mathrm{b}$ or $\mathrm{r}, \mathrm{b}$ quark degrees of freedom. It allows us to construct field distribution between three quarks similar to the fig. 3 .

The construction of the field distribution of a nonabelian gauge fields between three quarks pre- sented here is approximate one. More exact description one can obtain considering the quarks spaced at a finite distance. Such construction can be considered as a model of a nucleon filled with three quarks. The model considered here is a rough approximation for nucleon.

\section{Acknowledgements}

This work was partially supported by grants No. 139 in fundamental research in natural sciences by the Science Committee of the Ministry of Education and Science of Kazakhstan.

\section{References}

[1] R. Alkofer and L. von Smekal, The infrared behavior of QCD Green's functions: Confinement, dynamical symmetry breaking, and hadrons as relativistic bound states. // Phys. Rept. - 2001. - Vol. 353. - P. 281. [arXiv:hep-ph/0007355].

[2] Dzhunushaliev V.SU(3) flux tube gluon condensate // arXiv:1010.1621v3 [hep-ph]. - 2013.

[3] BornyakovV. G., Polikarpov M. I., Chernodub M. N., Suzuki T. and Schierholz G.Color confinement and hadron structure in lattice chromodynamics // Phys. Usp. -2004. - Vol. 47. - P. 17. [Usp. Fiz. Nauk. - 2004. Vol. 47. - P. 19].

[4] Heisenberg W., Introduction to the unified field theory of elementary particles. - London, NY etc.: Interscience, 1966. - $192 \mathrm{p}$.

[5] Greiner W., Schramm S., Stein E. Quantum chromodynamics. - Berlin, Heidelberg, NY: Springer, 2002. $572 \mathrm{p}$. 